\title{
In vivo overexpression of X-linked inhibitor of apoptosis protein protects against neomycin-induced hair cell loss in the apical turn of the cochlea during the ototoxic-sensitive period
}

\author{
Shan Sun ${ }^{1+}$, Mingzhi Sun ${ }^{2+}$, Yanping Zhang ${ }^{1}$, Cheng Cheng $^{3}$, Muhammad Waqas ${ }^{3}$, Huiqian Yu ${ }^{2}$, \\ Yingzi He ${ }^{1}$, Bo Xu ${ }^{4}$, Lei Wang ${ }^{5,6}$, Jian Wang ${ }^{7}$, Shankai Yin ${ }^{7}$, Renjie Chai ${ }^{3 *}$ and Huawei Li $^{2,5,8 *}$ \\ ${ }^{1}$ Research Center, Affiliated Eye and ENT Hospital of Fudan University, Shanghai, China \\ ${ }^{2}$ Department of Otorhinolaryngology, Affiliated Eye and ENT Hospital of Fudan University, Shanghai, China \\ ${ }^{3}$ Key Laboratory for Developmental Genes and Human Disease, Ministry of Education, Institute of Life Sciences, Southeast University, Nanjing, China \\ ${ }^{4}$ Anesthesiology Department, Xin Hua Hospital Affiliated to Shanghai Jiao Tong University School of Medicine, Shanghai, China \\ ${ }^{5}$ Institute of Stem Cell and Regeneration Medicine, Institutions of Biomedical Science, Fudan University, Shanghai, China \\ ${ }^{6}$ State Key Laboratory of Genetic Engineering, MOE Key Laboratory of Contemporary Anthropology, School of Life Sciences, Fudan University, Shanghai, China \\ 7 Department of Otolaryngology, The Sixth Hospital Affiliated to Shanghai Jiao Tong University, Shanghai, China \\ ${ }^{8}$ State Key Laboratory of Medical Neurobiology, Fudan University, Shanghai, China
}

\section{Edited by:}

Michael E. Smith, Western Kentucky University, USA

Reviewed by:

Ping Liu, University of Connecticut Health Center, USA

Hailan Yao, Roskamp Institute, USA

\section{*Correspondence:}

Renjie Chai, Key Laboratory for

Developmental Genes and Human

Disease, Ministry of Education,

Institute of Life Sciences, Southeast

University, Room North 301 ,

WenzhengLi Building, No. 2,

Sipailou Road, Xuanwu District,

Nanjing 210096, China

e-mail: renjiec@seu.edu.cn;

Huawei Li, Department of

Otorhinolaryngology, Affiliated Eye and ENT Hospital of Fudan

University, Room 611, Building 9,

No. 83, Fenyang Road, Xuhui

District, Shanghai 200031, China

e-mail: hwli@shmu.edu.cn

tThese authors have contributed equally to this work.
Aminoglycoside-induced cochlear ototoxicity causes hair cell $(\mathrm{HC})$ loss and results in hearing impairment in patients. Previous studies have developed the concept of an ototoxicity-sensitive period during which the cochleae of young mice are more vulnerable to auditory trauma than adults. Here, we compared neomycin-induced ototoxicity at the following four developmental ages in mice: postnatal day (P)1-P7, P8-P14, P15-P21, and P60-P66. We found that when neomycin was administered between P8 and P14, the auditory brainstem response threshold increase was significantly higher at low frequencies and $\mathrm{HC}$ loss was significantly greater in the apical turn of the cochlea compared to neomycin administration during the other age ranges. Quantitative real-time PCR (qPCR) data revealed that the expression of apoptotic markers, including Casp3 and Casp9, was significantly higher when neomycin was injected from P8 to P14, while the expression of the X-linked inhibitor of apoptosis protein (XIAP) gene was significantly higher when neomycin was injected from P60 to P66. Because XIAP expression was low during the neomycin-sensitive period, we overexpressed XIAP in mice and found that it could protect against neomycin-induced hearing loss at low frequencies and $\mathrm{HC}$ loss in the apical turn of the cochlea. Altogether, our findings demonstrate a protective role for $\mathrm{XIAP}$ against neomycin-induced hearing loss and $\mathrm{HC}$ loss in the apical turn of the cochlea during the ototoxic-sensitive period, and suggest that apoptotic factors mediate the effect of neomycin during the ototoxic-sensitive period.

Keywords: caspase, apoptosis, IAP, XIAP, aminoglycosides, hair cell loss, hearing loss

\section{INTRODUCTION}

Aminoglycosides can be ototoxic when administered to adults, children, and infants. It has been reported that the incidence of aminoglycoside-induced cochlear ototoxicity in neonates is greater than that in adults. In addition, the risk of ototoxicity is higher in preterm neonates than in full-term neonates (Henley and Rybak, 1993). The concept of an ototoxicitysensitive period was first proposed in the 1980s (Chen and Aberdeen, 1981; Chen and Saunders, 1983; Eggermont, 1986) as a time period during which the cochleae of young animals are

Abbreviations: HC, hair cell; $\mathrm{P}$, postnatal day; WT, wild type; ABR, auditory brainstem response; XIAP, X-linked inhibitor of apoptosis protein; IAP, inhibitor of apoptosis protein; Xafl, XIAP-associated factor 1; AIF, apoptosis-inducing factor; BIR, baculoviral IAP repeat. more vulnerable to auditory traumas (Eggermont, 1986; Henley and Rybak, 1995; Henley et al., 1996). However, the mechanisms involved in the ototoxicity-sensitive period are not well understood.

Several hypotheses have been proposed to describe the hypersensitivity of neonates to ototoxic drugs, and caspase-mediated apoptosis is a common theme (Forge and Li, 2000; Matsui et al., 2002). As one of the primary apoptosis executioner molecules (Ashkenazi and Dixit, 1998; Debatin and Krammer, 2004; Jiang and Wang, 2004; Salvesen and Riedl, 2008), caspase-3 has been widely used to assess the apoptosis of hair cells (HCs) in aminoglycoside-induced ototoxicity (Forge, 1985; Nakagawa et al., 1997; Cunningham et al., 2002; Wei et al., 2005; Tabuchi et al., 2007). Thus, caspase inhibition as a method to prevent 
cochlear HC death might be a useful treatment strategy for aminoglycoside-induced ototoxicity.

Inhibitor of apoptosis protein (IAP) prevents apoptosis by blocking the classic caspase-mediated apoptotic cascade and the JNK pathway. X-linked inhibitor of apoptosis protein (XIAP) is the most potent IAP and is a broad-range suppressor of apoptosis that functions by directly inhibiting caspases (Deveraux and Reed, 1999; Deveraux et al., 1999). XIAP is broadly expressed in all human tissues except peripheral blood leukocytes, and XIAP overexpression increases the survival of many cell types upon exposure to a variety of apoptotic triggers (Emamaullee et al., 2005; Zhu et al., 2007; Hu et al., 2010; Plesner et al., 2010; Wang et al., 2010, 2011; Unsain et al., 2013).

In this study, we first compared neomycin-induced hearing loss and $\mathrm{HC}$ loss at different developmental stages in mice. We then measured the expression levels of apoptosis-related genes in response to neomycin administration in mice at different developmental stages. Finally, we used XIAP overexpression mice to investigate the mechanisms through which XIAP and downstream apoptotic factors affect neomycin-induced ototoxicity during the sensitive period.

\section{MATERIALS AND METHODS MOUSE MODELS AND TREATMENTS}

Transgenic mice overexpressing XIAP (Wang et al., 2010, 2011) were kind gifts from Dr. Robert G. Korneluk at the Children's Hospital of Eastern Ontario Research Institute (Ottawa, Ontario, Canada). Experiments were performed in C57BL/6J wild-type (WT) mice and in XIAP overexpression mice (Experimental Animal Center, Shanghai Medical College of Fudan University, China). Postnatal day (P)0 was defined as the day of birth. Mice received a daily subcutaneous injection of neomycin $(200 \mathrm{mg} / \mathrm{kg})$ or sterile saline for 7 days. All animal procedures were performed according to protocols approved by the Animal Care and Use Committee of Fudan University and were consistent with the National Institutes of Health Guide for the Care and Use of Laboratory Animals. All efforts were made to minimize the number of animals used and prevent their suffering.

\section{AUDITORY BRAINSTEM RESPONSE (ABR) TEST}

The hearing thresholds of the mice were examined with the $\mathrm{ABR}$ test. In this test, changes in the electrical activity of the brain in response to sound were recorded via electrodes that were placed on the scalp of the mice. Animals were anesthetized with ketamine $(100 \mathrm{mg} / \mathrm{kg})$ and xylazine $(25 \mathrm{mg} / \mathrm{kg})$ and placed on a thermostatic heating pad in a sound-attenuating chamber to maintain their body temperatures at $38^{\circ} \mathrm{C}$. Frequencyspecific auditory responses were measured using the Tucker-Davis Technology system (TDT System III, Alachua, FL, USA) as previously described (Wang et al., 2010). All ABR tests were performed on mice older than P21.

\section{TISSUE PREPARATION}

After sacrificing the mice, the right otic capsule was immediately isolated, rapidly frozen in liquid nitrogen, and stored at $-70^{\circ} \mathrm{C}$ until further processing. To obtain the total RNA and protein extract, 10 cochleae were pooled in ice-cold buffer and processed with the Qiagen AllPrep ${ }^{\circledR}$ DNA/RNA/Protein Mini kit following the manufacturer's instructions. The RNA concentration was measured with a Bio-Rad spectrophotometer. Complementary (c)DNA was synthesized from $1 \mu \mathrm{g}$ total RNA by reverse transcription using random primers (Promega) and Superscript III reverse transcriptase (Life Technologies, Foster City, CA, USA) following the manufacturer's protocols. Quantitative real-time PCR (qPCR) was performed using SYBR Green PCR Master Mix (Life Technologies) on a Bio-Rad IQ5 Detection System (Bio-Rad, USA). Primer sequences are listed in Table 1. The left temporal bones were collected and stored overnight in $4 \%$ paraformaldehyde before decalcification in EDTA [4\% in phosphate-buffered saline (PBS), $\mathrm{pH}=6.4$ ] for a total of $72 \mathrm{~h}$. The otic capsule was then removed, and the cochlea was carefully isolated from the surrounding bony tissue. The organ of Corti was separated and immediately processed for immunofluorescent staining.

\section{IMMUNOFLUORESCENCE}

After fixation, cochlear samples were blocked with 10\% normal donkey serum in $10 \mathrm{mM}$ PBS ( $\mathrm{pH} 7.4$ ) with $0.3 \%$ Triton-X100 for $1 \mathrm{~h}$ at room temperature (RT) and then incubated with primary antibody overnight at $4^{\circ} \mathrm{C}$. The next day the tissues were incubated for $2 \mathrm{~h}$ at $4^{\circ} \mathrm{C}$ with 488 - or 594-conjugated donkey secondary antibody (1:500 dilution, Invitrogen) and 4,6-diamidino2-phenylindole (DAPI, 1:800 dilution, Sigma-Aldrich). Omission of primary antibody served as the negative control. The following primary antibodies were used: anti-myosin VIIA (myo7a) (1:500 dilution, cat. \#25-6790 Proteus BioSciences), and anticleaved caspase-3 (1:1000 dilution, cat. \#9579S, Cell Signaling Technology).

Cochleae were dissected into apical, middle, and basal turns and images were taken using a Leica SP5 confocal fluorescence microscope (Leica, Germany).

Table 1 | PCR primer sequences used in the experiments.

\begin{tabular}{|c|c|c|}
\hline Gene & Forward sequence & Reverse sequence \\
\hline Diablo NM023232 & 5'-CTGGGGGCCCTGGGGTCCTA-3' & 5'-GAAAACTGGTCCCTGGTGGTCA-3' \\
\hline Htra2 NM 019752 & 5'-AGTAGGGCGGGCGAGGAGAGT-3' & 5'-AGCAGAGCCCGGAGGTCAGG-3' \\
\hline Casp3 NM009810 & 5'-CGGGGTACGGAGCTGGACTGT-3' & 5'-ATGCTGCAAAGGGACTGGATGAAC-3' \\
\hline Casp 9 NM 015733 & 5'-AGGCCCGTGGACATTGGTTCT-3' & 5'-AGTTGGAGCCCGTGCGTGTG-3' \\
\hline
\end{tabular}




\section{CELL COUNTS}

For HC quantification in the neomycin-treated samples, we imaged the entire cochlea using a $63 \times$ objective and counted the myo7a+ HCs that remained. The same procedure was used to quantify caspase- $3+$ and caspase- $3+/$ myo $7 a+$ cells. For all experiments, only one cochlea from each mouse was used for immunofluorescence and quantification. Thus, $n$ represents the number of mice examined.

\section{STATISTICAL ANALYSES}

Data were expressed as mean $\pm \mathrm{SD}$. ABR thresholds were analyzed by a two-way ANOVA followed by a Newman-Keuls post-hoc test. Immunofluorescence analysis was performed with a two-tailed, unpaired Student's $t$-test when comparing two groups or with a one-way ANOVA followed by a Dunnett's multiple comparisons test when comparing more than two groups. $p<0.05$ was considered statistically significant.

\section{RESULTS}

\section{NEOMYCIN TREATMENT AT DIFFERENT STAGES INDUCED DIFFERENT} LEVELS OF HEARING LOSS

Aminoglycoside-induced cochlear toxicity in neonatal mice is greater than that in adults (Chen and Aberdeen, 1981; Chen and Saunders, 1983). Here we divided the WT mice into four groups for daily subcutaneous injections of neomycin $(200 \mathrm{mg} / \mathrm{kg})$ or saline. Group 1 was injected from P1 to P7, Group 2 was injected from P8 to P14, Group 3 was injected from P15 to P21, and Group 4 was injected from P60 to P66. We measured hearing function using pure tone $\mathrm{ABR}$ thresholds in the neomycin-treated and control mice 2 weeks after the last injection. At low frequencies, such as $8 \mathrm{kHz}$ and $16 \mathrm{kHz}, \mathrm{ABR}$ thresholds were significantly increased in neomycin-treated mice compared to controls for Groups 2 and 3 ( $p<0.01$ for both frequencies and both groups; $n=6-7)$. However there was no significant difference between the neomycin-treated mice and controls for Groups 1 and $4(p=0.194$ and $p=0.056$ for 8 and $16 \mathrm{kHz}$, respectively, in Group 1 and $p=0.218$ and $p=0.145$, respectively, in Group $4 ; n=6-7$ mice). At high frequencies, such as 24 and $32 \mathrm{kHz}$, ABR thresholds were significantly increased in all four groups of neomycin-treated mice compared to controls $(p<0.01$, $n=6-7$ ) (Figures 1A1-A4). Among all four groups, the increase in hearing thresholds in the neomycin-treated mice compared to controls was greatest in Group 2. At $8 \mathrm{kHz}$, the ABR thresholds in Group 2 increased significantly more than any other group ( $p<0.01, n=6-7)$; at $16 \mathrm{kHz}$, Groups 2 and 3 increased more than the other two groups $(p<0.01$ for both groups, $n=6-7)$; and at $24 \mathrm{kHz}$ and $32 \mathrm{kHz}$, Groups 1-3 increased more than Group $4(p<0.01, n=6-7)$ (Figure 1B).

\section{NEOMYCIN TREATMENT AT DIFFERENT STAGES INDUCED DIFFERENT LEVELS OF HC LOSS}

To further investigate the neomycin-induced ototoxicity in the four groups, HC loss in all three turns of the cochlea was evaluated in 3-5 cochleae from each group. Samples were compared 2 weeks after the last injection of neomycin or saline (Figure 2). We found that the number of remaining HCs in Group 2 was significantly lower in all three turns compared to all other groups $(p<0.01, n=3-5)$. In the apical turn, there was no significant difference in the number of remaining HCs among Groups 1, 4, and saline controls $(n=3-5)$. In the middle and basal turns, the number of remaining HCs in Groups 2 and 3 was significantly lower than Groups 1, 4, and saline controls $(p<0.01$ for both groups, $n=3-5$ ). In the basal turn, the number of remaining HCs in Groups 1 and 4 were significantly lower than the saline control group ( $p<0.01$ for both groups, $n=3-5$ ) (Figure 2B).

These results showed that Groups 2 and 3 had significant HC loss in the apical turn-while the other groups had no significant $\mathrm{HC}$ loss - and that Group 2 in particular had significantly more $\mathrm{HC}$ loss in the middle and basal turns than all other groups. This also suggests that $\mathrm{HC}$ loss in the apical turn of Group 2 was caused by the hypersensitivity to neomycin-induced ototoxicity during the sensitive period.

\section{GENE EXPRESSION LEVELS OF APOPTOSIS FACTORS WERE DIFFERENT WHEN NEOMYCIN TREATMENT OCCURRED AT DIFFERENT AGES}

To further understand the mechanism resulting in the sensitive period for neomycin-induced ototoxicity, we investigated the gene expression of apoptosis-related genes by qPCR. In this set of experiments, we divided the WT mice into four groups as above and the mice were injected with neomycin or saline for 7 days and sacrificed 3 days after the last injection. The day of sacrifice was determined by cleaved-caspase- 3 expression in the cochlea as shown by the immunofluorescence in Figure S1A. The number of caspase-3+ cells reached its peak at 3 days after the last injection (Figure 4B1). Because the ABR and $\mathrm{HC}$ quantification results showed differences between apical and basal turns, we separated the cochlea into two parts, the apical $50 \%$ and the basal $50 \%$. We measured the gene expression of the following anti-apoptotic genes: Xiap (X-linked inhibitor of apoptosis), which belongs to a family of apoptotic suppressor proteins, and Xaf1 (XIAP associated factor 1), which binds to and counteracts the inhibitory effect of a member of the IAP (inhibitor of apoptosis) protein family. We also measured the expression levels of the following pro-apoptotic genes: Casp3 and Casp9, which are members of the caspase family and play central roles in the execution-phase of apoptosis; Diablo, which encodes an IAP-binding protein; and Htra2, which encodes a serine protease that induces apoptosis by binding to the apoptosis inhibitory protein XIAP. All of the gene expression data were normalized to the gene expression of P3 cochleae treated with saline. When compared to saline controls, we found that the expression levels of the anti-apoptotic genes Xiap and Xaf1 were significantly increased in neomycin-treated cochleae from Groups 3 and 4 ( $p<0.01$ for both groups, $n=3$ ) and that the expression levels in the apical $50 \%$ of the cochlea were slightly higher than in the basal 50\%. There was no significant increase of Xiap or Xaf1 in neomycin-treated cochleae in Groups 1 and 2 compared to their saline controls (Figure 3A), and this indicated that Xiap might protect the cochlea from neomycin-induced ototoxicity. Compared to saline controls, the expression levels of the apoptotic genes Casp3, Casp9, Diablo, and Htra2 were significantly increased in neomycin-treated cochleae in Groups 2 and 3 ( $p<0.01$ for both groups, $n=3$ ). The expression of Casp3 was also significantly increased in Groups 1 and $4(p<0.01$ for both groups, $n=3)$, but the expression levels 


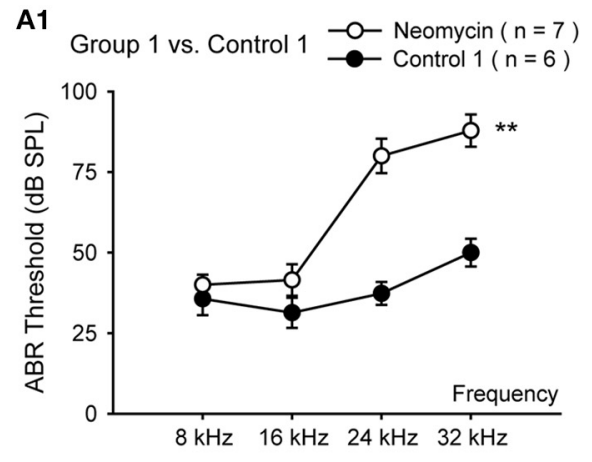

A3

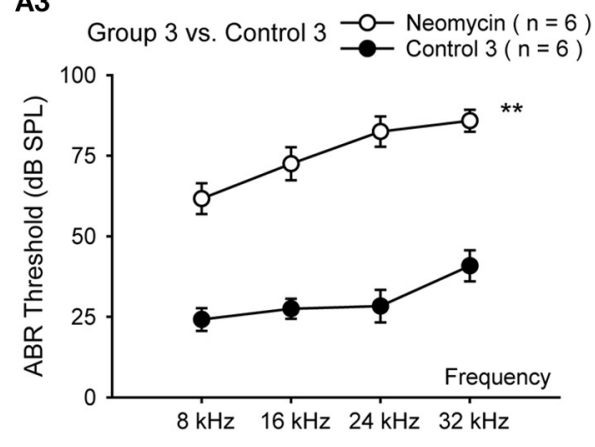

B

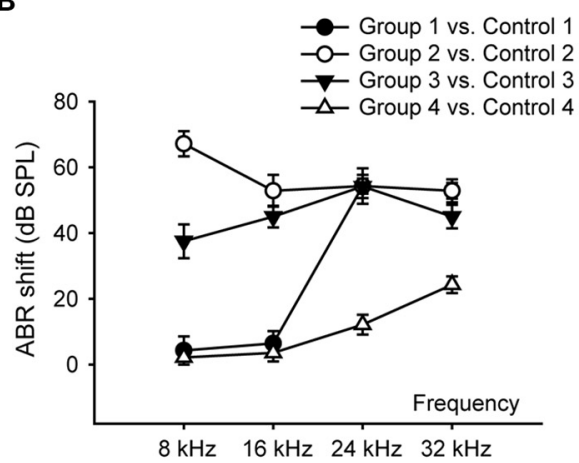

FIGURE 1 | Hearing loss in neomycin-treated WT mice. (A) ABR

measurements of WT mice 2 weeks after the last injection of neomycin or

saline (control). Neomycin or saline was injected once daily between P1 and

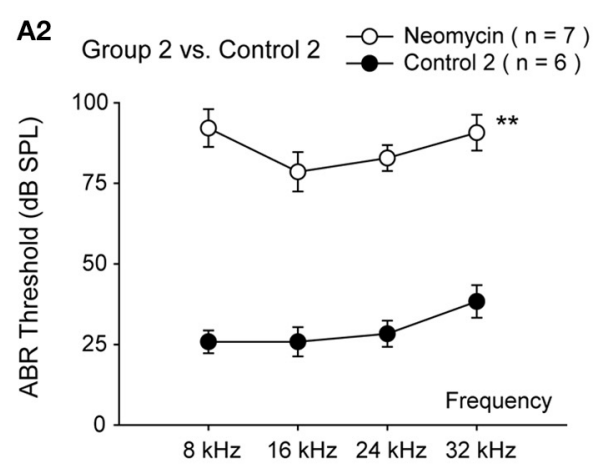

A4

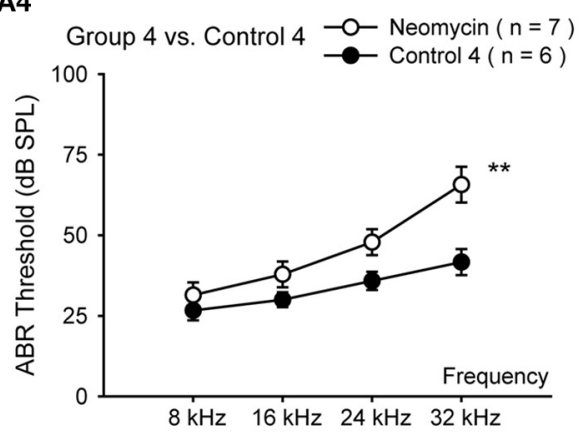

P7 (Group 1) (A1), P8 and P14 (Group 2) (A2), P15 and P21 (Group 3) (A3), or P60 and P66 (Group 4) (A4). (B) ABR threshold shifts of neomycin-treated samples compared to control. ${ }^{* *} p<0.01$ vs. control, $n=6-7$. of the other three apoptotic genes were only slightly increased in Groups 1 and 4. In addition, the expression of apoptotic genes in the apical $50 \%$ of the cochlea was slightly lower than the basal 50\% (Figure 3B). When compared among the four groups, we found that the expression levels of the anti-apoptotic genes Xiap and Xaf1 increased with age in saline controls. After neomycin exposure, Xiap and Xafl expression levels in Groups 1 and 2 were similar to controls. In Group 4, however, the expression levels of Xiap and Xaf1 were significantly higher than all other groups (Figure 3A). Because XIAP has protective effects against apoptosis, one underlying cause of the sensitive period for neomycin-induced ototoxicity might be low XIAP levels. We also found that the expression levels of pro-apoptotic genes Casp3,
Casp9, Diablo, and Htra2 were significantly higher in Group 2in which neomycin was injected during the ototoxicity-sensitive period of P8 to P14 - than in all other groups (Figure 3B), and this suggests that apoptotic factors are involved in the sensitive period for neomycin-induced ototoxicity.

\section{CASPASE-MEDIATED APOPTOSIS WAS INVOLVED IN THE SENSITIVE PERIOD OF NEOMYCIN-INDUCED OTOTOXICITY}

To confirm the involvement of caspase-mediated apoptosis in the postnatal sensitive period for neomycin-induced ototoxicity in vivo, we also divided the WT mice into four groups as described above, injected them with neomycin or saline for 7 days, sacrificed the mice at 1,3, 5, or 7 days after the last injection, and 


\section{A Myosin7a/DAPI}
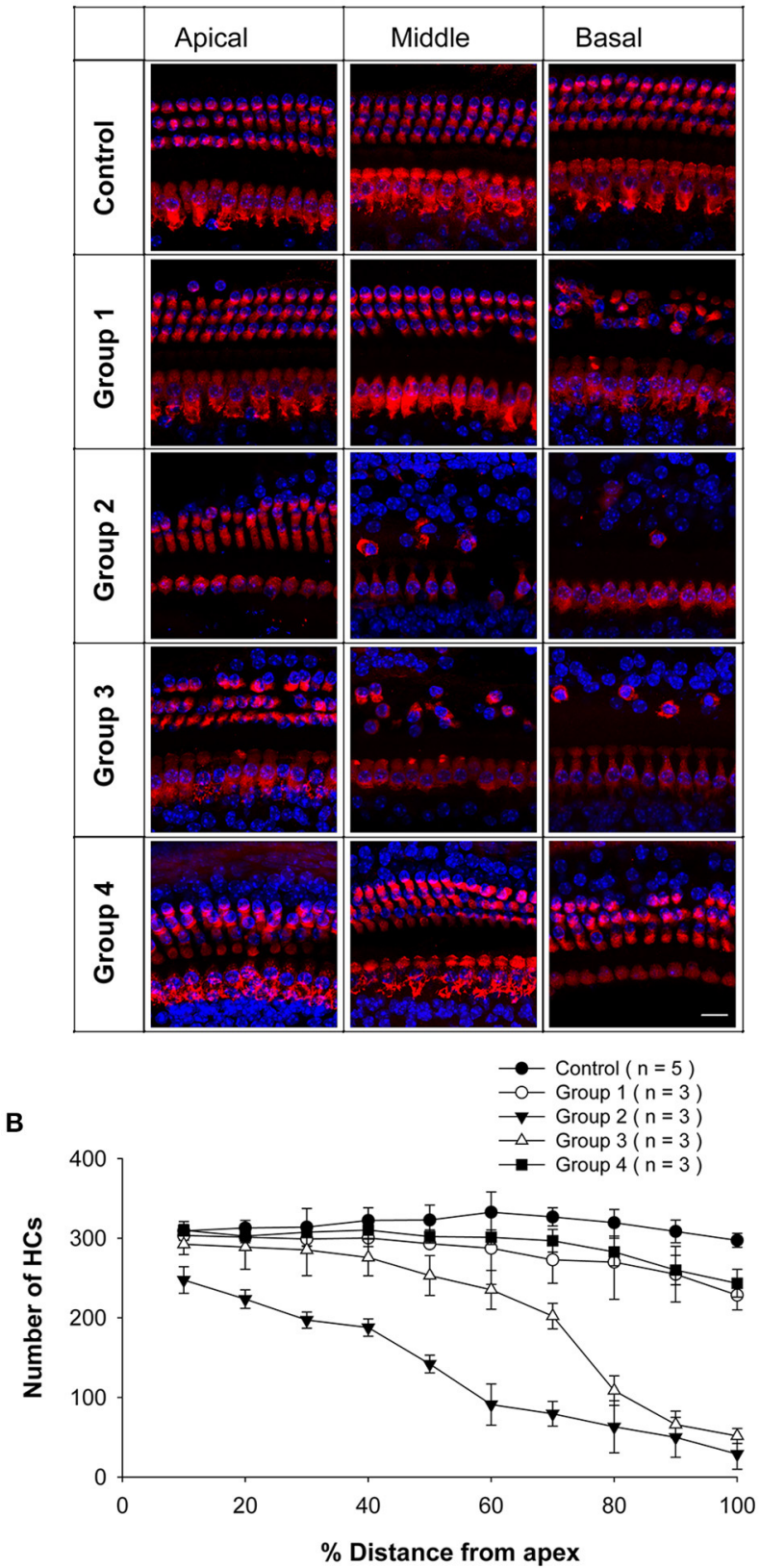

FIGURE 2 | HC loss in neomycin-treated WT mice. (A) Representative confocal images of myo7a immunofluorescence in cochlear whole mounts of WT mice treated with neomycin or saline (control) once daily between P1 and P7 (Group 1), P8 and P14 (Group 2), P15 and P21 (Group 3), or P60 and P66 (Group 4) and sacrificed 2 weeks after the last injection of neomycin or saline. (B) Myo7a+ HC quantification in WT mice treated with neomycin or saline (control) and sacrificed 2 weeks after the last injection of neomycin or saline. Scale Bar in $\mathbf{A}=10 \mu \mathrm{m}$.

stained the cochleae with cleaved-caspase- 3 and myo7a antibodies (Figure S1A). We found that the number of HCs kept decreasing from day 1 to 7 in all neomycin-treated groups. At each time point, there was a significant difference between Group 2 and the three other neomycin-treated groups $(p<0.01$, Figure S1). Moreover, the number of caspase- $3+$ cells was greatest at 3 days following the neomycin injection (Figures 4B1, S1A), and this was the time-point chosen for further analysis. At 3 days after neomycin or saline treatment, no caspase-3+ cells were observed in any of the three turns of the control cochleae treated with saline, and this was consistent with a previous report (Kaiser et al., 2008). Among the four groups with neomycin treatment, Group 2 had significantly more caspase $-3+$ and caspase $-3+/$ myo $7 \mathrm{a}+$ cells in the apical turn of cochlea $(17.00 \pm 4.58$ cells and $7.67 \pm 2.10$ cells per cochlear apical turn, respectively, $p<0.01, n=3)$. The other three groups had very few caspase- $3+$ cells and almost no caspase-3+/myo7a + cells in the apical turn (Figures 4A,B2,B3). This result suggested that caspase-mediated apoptosis is involved in the neomycin-induced ototoxicity of the apical turn during the sensitive period. In middle and basal turns, all four groups had significantly more caspase $-3+$ and caspase $-3+/$ myo $7 a+$ cells than the control groups $(p<0.01, n=3)$, but Group 2 had significantly more caspase- $3+$ cells $(31.67 \pm 3.51$ cells and $18.33 \pm 1.38$ cells per cochlear middle and basal turn, respectively) compared to Groups 1,3 , and 4 (17.33 \pm 5.86 cells and $10.67 \pm 0.58$ cells, $17.33 \pm 3.52$ cells and $11.33 \pm 2.31$ cells, $8.33 \pm 1.52$ cells and $4.00 \pm 2.13$ cells per cochlear middle and basal turn per group, respectively, $p<0.01, n=3$ ) and Group 2 had more caspase$3+/$ myo7a + cells $(11.67 \pm 1.53$ cells and $5.67 \pm 1.53$ cells per cochlear middle and basal turn, respectively) compared to Groups 1,3 , and $4(3.00 \pm 1.12$ cells and $1.67 \pm 0.58$ cells, $2.67 \pm 0.58$ cells and $2.00 \pm 1.21$ cells, $2.00 \pm 0.67$ cells and $1.33 \pm 0.58$ cells per cochlear middle and basal turns per group, respectively, $p<0.01, n=3$ ) (Figures 4A,B2,B3). This result suggested that caspase-mediated apoptosis is also involved in the postnatal sensitive period of neomycin-induced ototoxicity in middle and basal turns.

\section{XIAP OVEREXPRESSION ATTENUATED NEOMYCIN-INDUCED HC LOSS IN THE APICAL TURN DURING THE OTOTOXICITY-SENSITIVE PERIOD}

To further investigate whether apoptotic factors are involved in the sensitive period to neomycin-induced ototoxicity in the apical turn, we took advantage of genetic methods to inhibit the apoptotic factors in neomycin-treated mice. Here we used transgenic XIAP overexpression mice, in which the coding sequence of XIAP is under the control of a ubiquitin promoter (ubXIAP) (Wang et al., 2010). In this experiment, we injected WT or ubXIAP overexpression mice with neomycin or saline during the ototoxicity-sensitive period (Group 2, P8-P14). ABR thresholds were measured from six mice in each group at $\mathrm{P} 30$, and $\mathrm{HC}$ loss in all three cochlear turns was evaluated from $4-5$ cochleae in each group at P30 (Figure 5A). In the neomycin-treated groups, ABR thresholds were significantly decreased at $8 \mathrm{kHz}$ in the XIAP overexpression mice compared to WT mice $(p<0.01, n=6)$. There was a slight decrease at $16 \mathrm{kHz}(p<0.05, n=6)$, but no significant difference was detected at 24 and $32 \mathrm{kHz}$. There was no $\mathrm{ABR}$ threshold increase in the XIAP overexpression mice without neomycin injection (Figure 5A2).

Moreover, in the neomycin-treated groups, there was almost no HC loss in the apical turn of the cochleae from XIAP overexpression mice, and this demonstrated that XIAP overexpression 

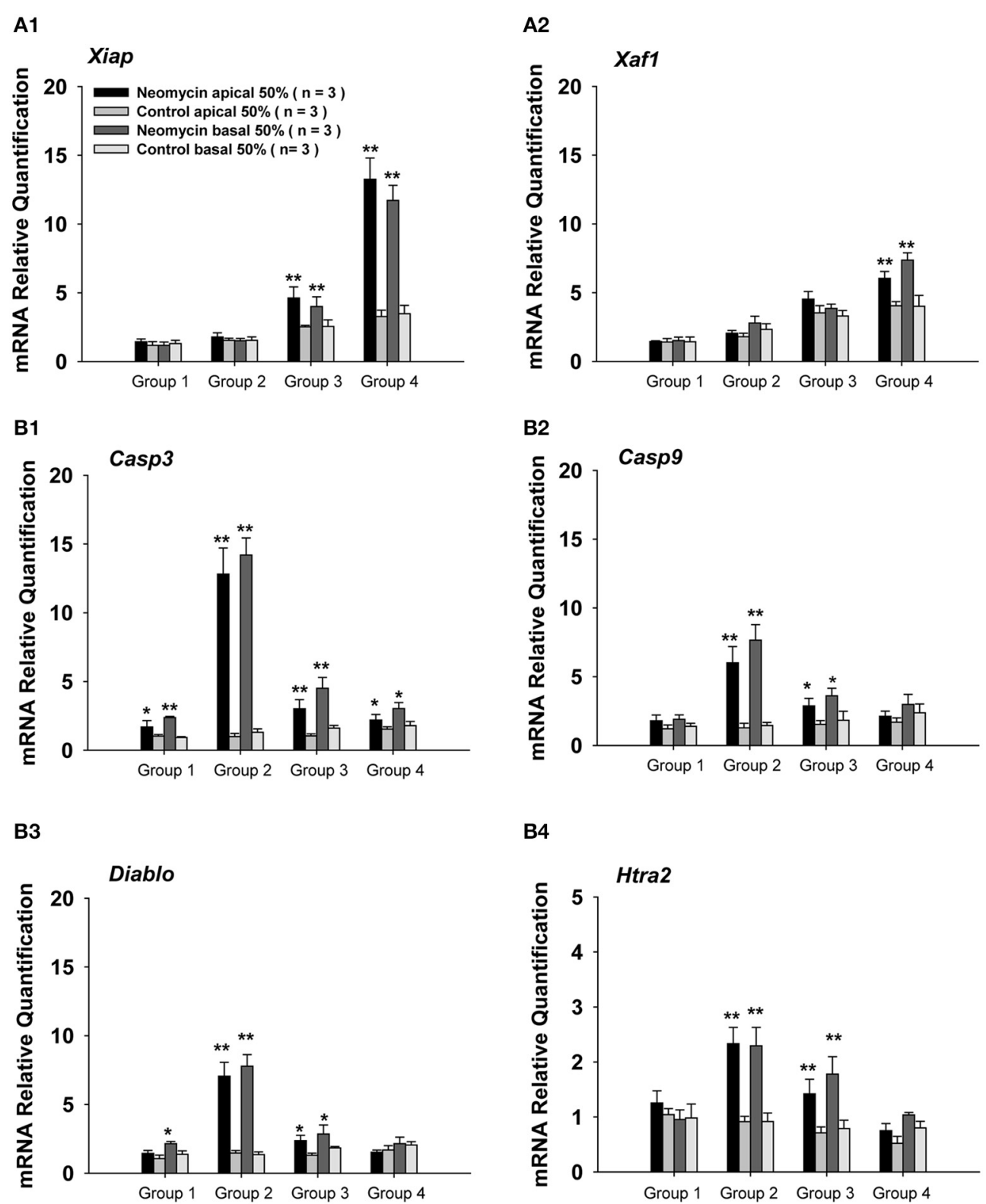

B4

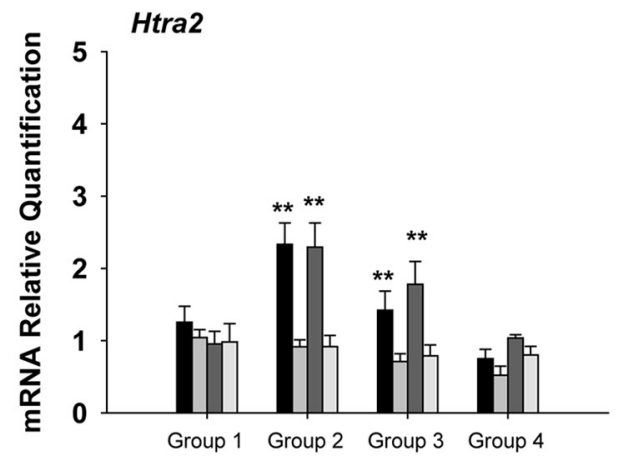

FIGURE 3 | Apoptosis-related gene expression in neomycin-treated WT mice. (A,B) mRNA expression of the anti-apoptotic genes Xiap (A1) and Xaf1 (A2) and the pro-apoptotic genes Casp3 (B1), Casp9 (B2), Diablo (B3), and Htra2 (B4) in the apical $50 \%$ and basal $50 \%$ of the cochleae in WT mice

sacrificed 3 days after the last injection of neomycin or saline (control). Neomycin or saline was injected between P1 and P7 (Group 1), P8 and P14 (Group 2), P15 and P21 (Group 3), or P60 and P66 (Group 4). * $p<0.05$, $* * p<0.01$ vs. control, $n=3$.

almost completely prevented the neomycin-induced HC loss in the apical turn during the ototoxicity-sensitive period. HC loss was slightly attenuated in middle and basal turns in XIAP overexpression mice. There was no HC loss in XIAP overexpression mice without neomycin treatment (Figures 5A1,A3).

We further examined the cleaved-caspase- 3 expression in neomycin-treated XIAP overexpressing mice. In this experiment, we injected WT or ubXIAP mice with neomycin or saline from P8 to P14, sacrificed the mice at 3 days after the last injection, and stained the cochleae with cleaved-caspase- 3 and myo7a antibodies (Figure 5B1). We found that when treated with neomycin, XIAP overexpression mice had only a very small number of caspase$3+$ cells and no caspase- $3+/$ myo $7 \mathrm{a}+\mathrm{HCs}$ in the apical turn. They also had significantly fewer caspase-3+ cells and caspase$3+/$ myo $7 \mathrm{a}+\mathrm{HCs}$ in the middle and basal turns compared to the WT mice (Figures 5B2,B3, $p<0.05, n=3$ ). Together, these results suggest that when neomycin was injected during the ototoxicity-sensitive period, HC loss in the apical turn was primarily caused by the hypersensitivity to neomycin-induced ototoxicity (Figure 2). XIAP overexpression significantly reduced hearing loss and $\mathrm{HC}$ loss in the apical turn, but only partially prevented the $\mathrm{HC}$ loss in the middle and basal turns (Figure 5). We 

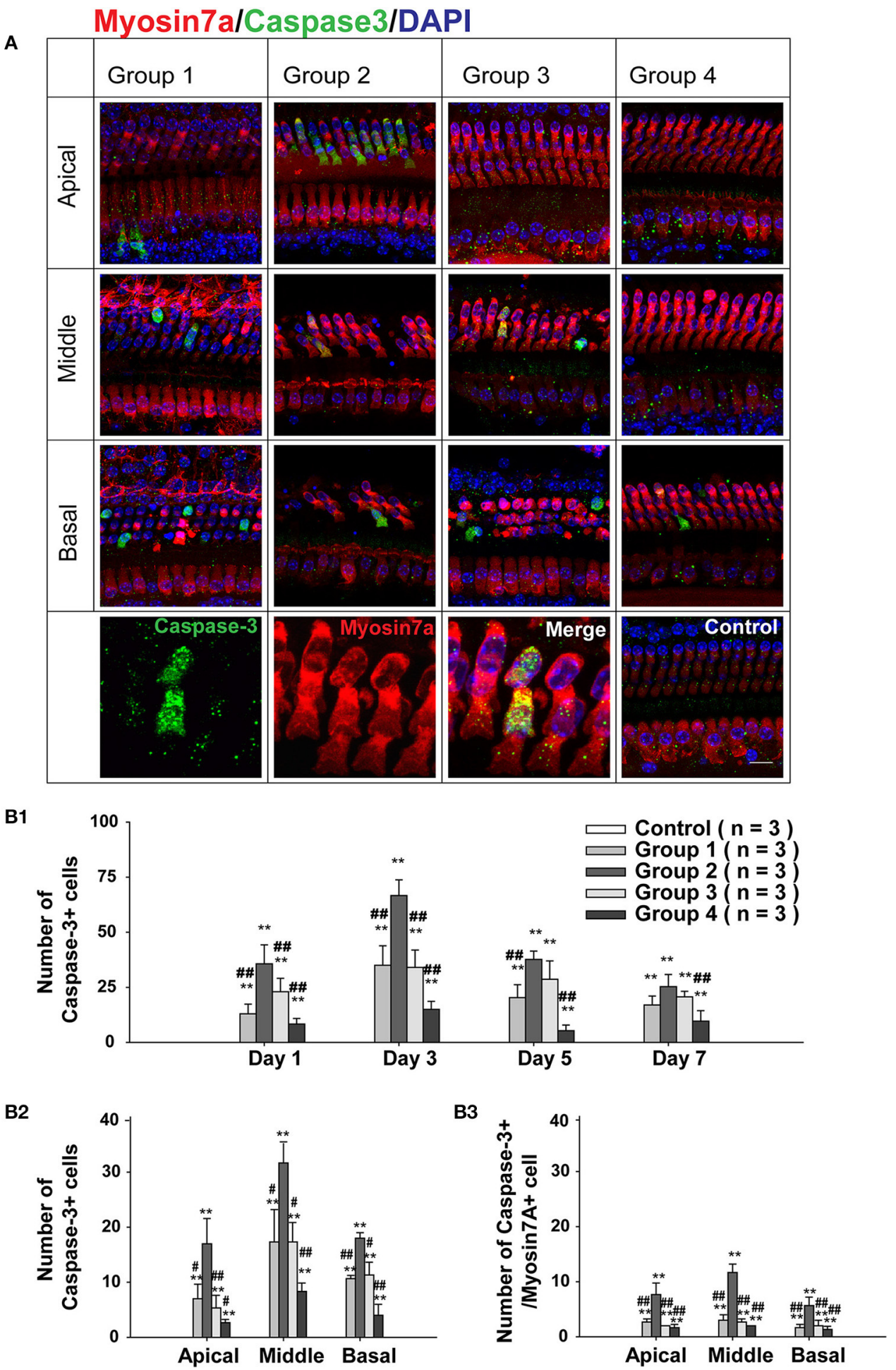

FIGURE 4 | Caspase-3 expression after neomycin treatment. (A)

Representative confocal images of all three turns of the cochlea stained with myo7a and cleaved caspase-3 in WT mice sacrificed 3 days after the last injection of neomycin or saline (control). Neomycin or saline was injected between P1 and P7 (Group 1), P8 and P14 (Group 2), P15 and P21 (Group 3), or P60 and P66 (Group 4). (B1) The number of caspase-3+ cells in the whole cochlea of WT mice treated with neomycin or saline (control) between P1 and P7 (Group 1), P8 and P14 (Group 2), P15 and P21 (Group
3), or P60 and P66 (Group 4) and sacrificed 1, 3, 5, or 7 days after neomycin or saline treatment. (B2,B3) The number of caspase-3+ cells (B2) and caspase- $3+/$ myo $7 a+$ cells (B3) in the three cochlear turns of WT mice treated with neomycin or saline (control) between P1 and P7 (Group 1), P8 and P14 (Group 2), P15 and P21 (Group 3), or P60 and P66 (Group 4) and sacrificed 3 days after the last injection of neomycin or saline treatment. ${ }^{* *} p<0.01$ vs. control and ${ }^{\#} p<0.05$, ${ }^{\# \#} p<0.01$, vs. Group $2, n=3$. Scale bar in $\mathbf{A}=10 \mu \mathrm{m}$ 
A1 Myosin7a/DAPI

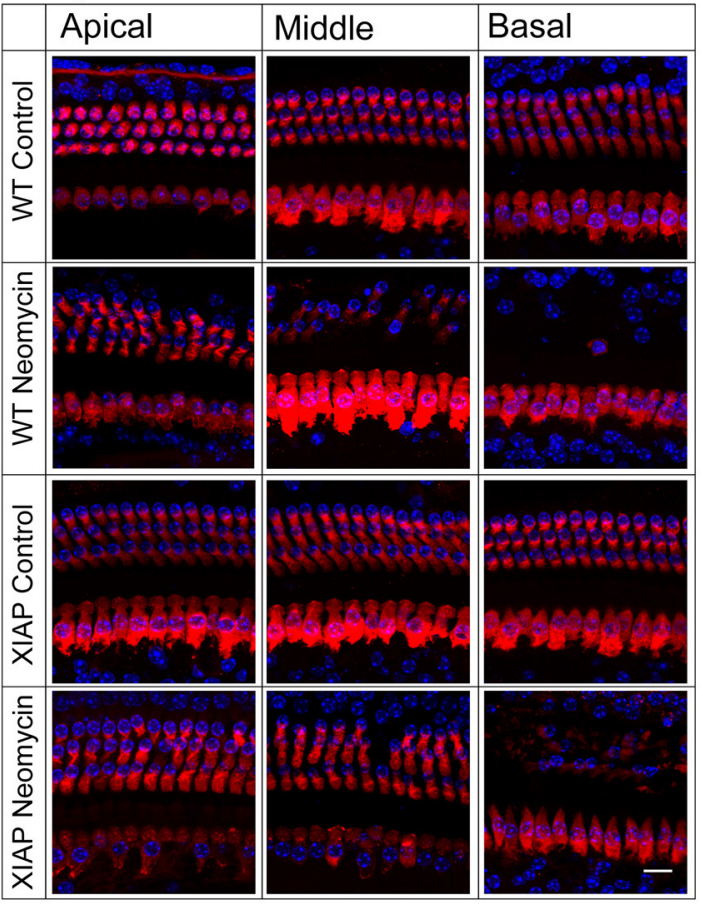

B1

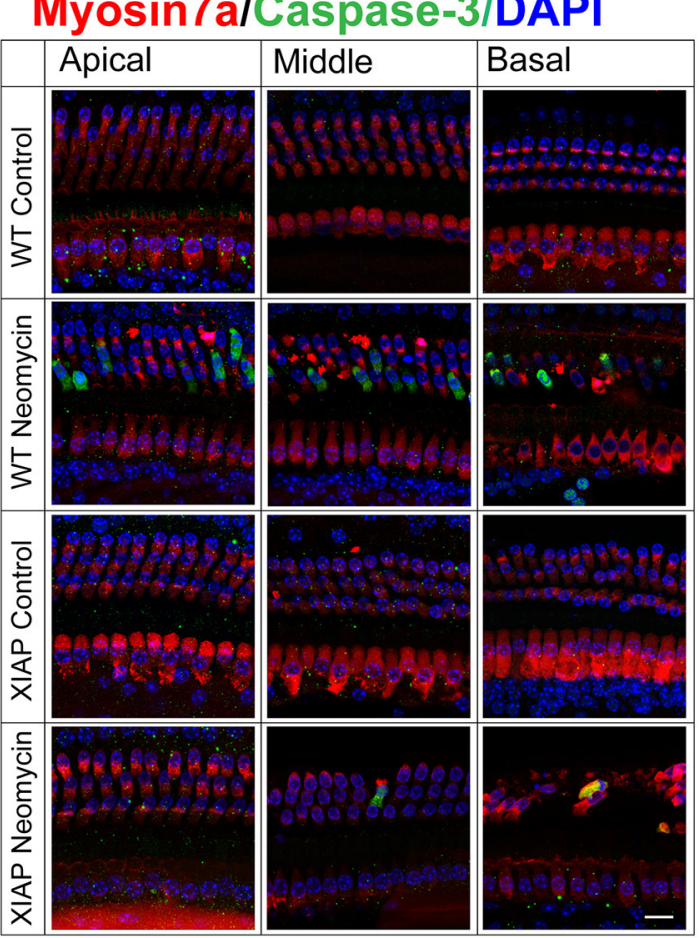

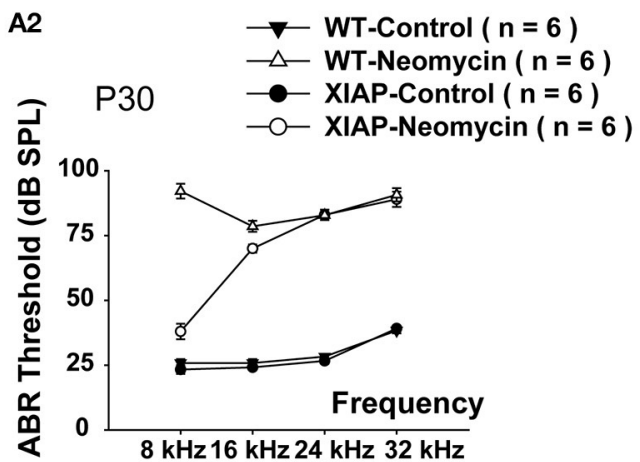

A3
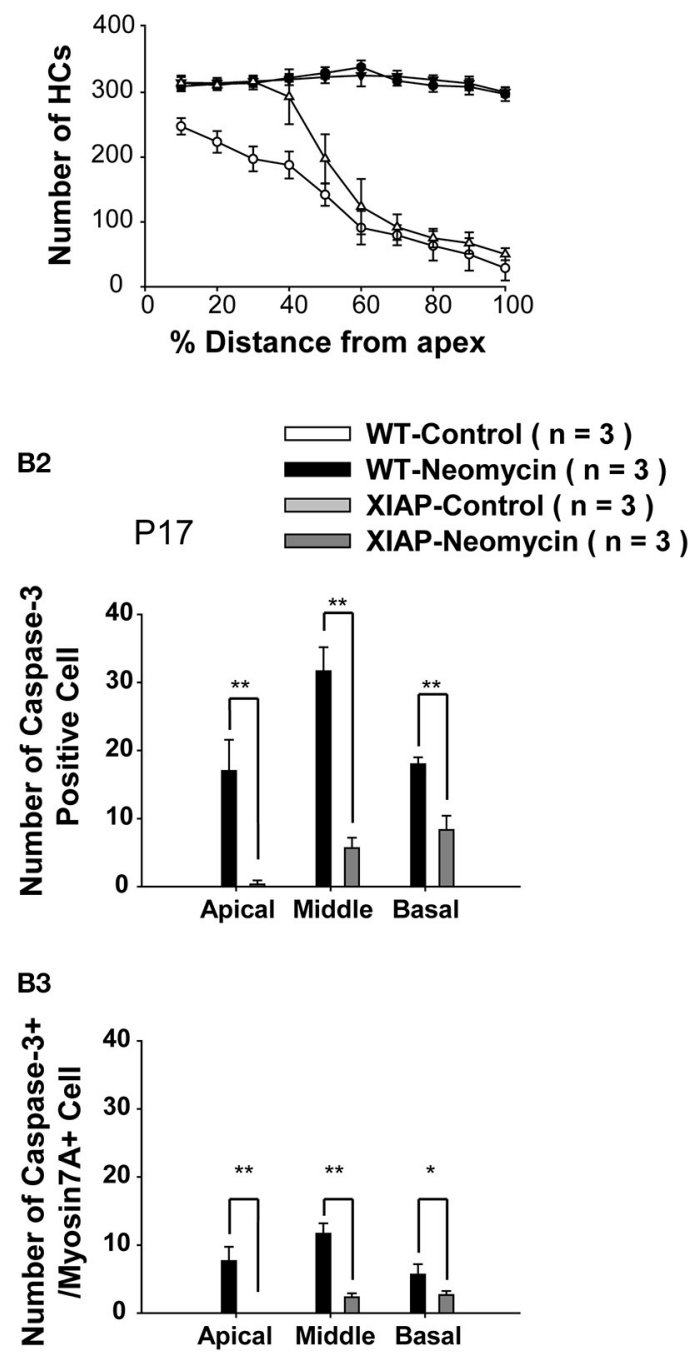

FIGURE 5 | Hearing loss and HC loss in neomycin-treated XIAP overexpression mice. (A1) Representative confocal images of myo7a immunofluorescence in cochlear whole mounts of XIAP overexpression and WT mice treated with neomycin or saline (control) between P8 and P14 (Group 2) and sacrificed at P30. ABR measurement (A2) and myo7a+ HC quantification (A3) of P30 XIAP overexpression and WT mice treated with neomycin or saline (control) between P8 and P14. (B1) Representative confocal images of myo7a and cleaved-caspase-3 immunofluorescence in cochlear whole mounts of XIAP overexpression and WT mice treated with neomycin or saline (control) between P8 and P14 (Group 2) and sacrificed at P17. (B2,B3) The quantification of caspase- $3+$ cells (B2) and caspase- $3+$ /myo7a + cells (B3) in the three cochlear turns of XIAP overexpression and WT mice treated with neomycin or saline (control) between P8 and P14 (Group 2) and sacrificed at P17. ${ }^{*} p<0.05,{ }^{* *} p<0.01$ vs. WT neomycin, $n=3$. Scale bar in $\mathbf{A}=10 \mu \mathrm{m}$. 
thus conclude that apoptotic factors are involved in the sensitive period of neomycin-induced ototoxicity in the apical turn of the cochlea.

\section{DISCUSSION}

Aminoglycosides are effective against aerobic gram-negative infections and are widely used to treat tuberculosis in people in developing countries and to treat life-threatening bacterial infections in premature infants across the world. All aminoglycosides have the potential for nephrotoxicity, ototoxicity, neuromuscular blockade, allergic reactions, and hematotoxicity, and these adverse effects limit the clinical use of aminoglycoside antibiotics. Aminoglycoside ototoxicity is typically associated with bilateral sensorineural hearing loss and tinnitus (Nadol, 1993), yet there have been only a few in vivo investigations of aminoglycoside ototoxicity in mammalian development.

Previous reports indicated that aminoglycoside-induced cochlear HC loss is more severe in neonatal mice than in adults, and this led to the development of the concept of an ototoxicitysensitive period (Chen and Aberdeen, 1981; Chen and Saunders, 1983; Eggermont, 1986; Henley and Rybak, 1993, 1995; Henley et al., 1996). However the mechanism underlying this sensitive period has not been elucidated. In our experiments, we injected neomycin during the four different ages of P1-P7, P8-P14, P15$\mathrm{P} 21$, and P60-P66 and found that all groups of C57BL/6J WT mice were sensitive to neomycin-induced hearing loss at high frequencies. However, at low frequencies only the P8-P14 group was sensitive to neomycin-induced hearing loss and only that group had significant HC loss in the apical turn. Taken together, these results suggest that $\mathrm{HC}$ loss in the apical turn is primarily caused by hypersensitivity to neomycin-induced ototoxicity during the sensitive period of $\mathrm{P} 8$ to $\mathrm{P} 14$.

Pharmacokinetics experiments have shown that aminoglycosides have a longer serum half-life and a lower serum clearance rate in neonates than in adults, and this might result in increased risk of permanent ototoxic damage in neonates (Henley and Rybak, 1993; Berard et al., 1994; Scaglione et al., 1995). In addition to metabolic and anatomic factors, animal models of aminoglycoside-induced ototoxicity have also indicated hypersensitivity of neonates to ototoxic drugs compared to adults, but the underlying mechanisms behind this hypersensitivity remain unclear. When aminoglycoside antibiotics are taken up into sensory HCs and transported to endosomes and lysosomes, lysosomal phospholipidosis is followed by lysosomal rupture and this lead to the release of aminoglycosides into the cytoplasm and impairment of mitochondrial respiration (Chen et al., 1996). This in turn can lead to several different biochemical events. (1) Complexes can form with transition metals (iron and copper), and these promote the formation of superoxide radicals such as hydroxyls. These in turn can trigger lipid peroxidation that progressively inhibits metabolic activities (Guo et al., 2001). (2) There is increased calcium influx into cells resulting in calcium overload. (3) Caspase-mediated apoptotic cascades are initiated (Forge, 1985; Li et al., 1995; Nakagawa et al., 1997; Cunningham et al., 2002). (4) JNK-mediated cell apoptosis is initiated (Ylikoski et al., 2002; Sugahara et al., 2006). (5) The permeability of the mitochondria changes, and this leads to the release of apoptosis-inducing factor and endonuclease G, both of which can then move into the nucleus and activate caspaseindependent pathways that can lead to apoptosis-like features in cells (Jiang et al., 2006).

In our study, HC damage in the basal turn of the cochlea was much more severe than in the apical turn, and this is correlated with previous reports that outer HCs from the basal turn are more susceptible to damage than outer HCs in the apical turn (Sha et al., 2001; Jensen-Smith et al., 2012). The underlying cause for this phenotype might be the following: (1) the cochlear blood flow moves more quickly in the basal turn and this results in higher concentrations of drugs in the basal turn; (2) the outer HCs of the basal turn are smaller and contain more mitochondria and are thus more susceptible to damage; and (3) the distribution and activity of drug transporters in basal and apical outer HCs is different (Ding and Salvi, 2007).

To investigate the underlying mechanism behind the hypersensitivity to neomycin-induced ototoxicity in the apical turn during the sensitive period, we measured the expression level of apoptosis-related genes when neomycin was injected during different periods. We found that the expression levels of the apoptotic factors Casp3, Casp9, Diablo, and Htra2 were significantly higher when neomycin was injected during the sensitive period compared to other periods, and this suggested that apoptotic factors are involved in the sensitive period for neomycin-induced ototoxicity.

We then investigated the possible molecular biological mechanism leading to the ototoxicity-sensitive period. XIAP has the most potent anti-apoptotic effects. It contains a single C-terminal RING domain and three tandem baculoviral IAP repeat (BIR) domains. BIR1 and BIR2 have been shown to inhibit caspase-3 and caspase-7 activation (Suzuki et al., 2001), and the caspase9 inhibitory activity of XIAP has been localized to the BIR3RING domain (Srinivasula et al., 2001; Shiozaki et al., 2003; Zou et al., 2003). It has been well established that overexpression of XIAP can protect against apoptosis. There is also evidence that AAV-mediated delivery of XIAP protects against cisplatin ototoxicity (Cooper et al., 2006). XIAP overexpression has been demonstrated to prevent neuronal death in models of transient cerebral ischemia and Parkinson's disease (Xu et al., 1999; Ishigaki et al., 2002; Crocker et al., 2003; Trapp et al., 2003). The same ubiquitous XIAP overexpression mice used in this study also exhibit later onset of presbycusis and an insensitivity to noise-induced hearing loss (Wang et al., 2010, 2011).

To further investigate the role of XIAP in the sensitive period for neomycin-induced ototoxicity, we injected neomycin into transgenic XIAP overexpression mice during the sensitive period. ABR data showed that XIAP overexpression protected against hearing loss from neomycin-induced ototoxicity at $8 \mathrm{kHz}$, but the protective effect was not significant at 16,24 , or $32 \mathrm{kHz}$. When we quantified the number of remaining HCs, we found that XIAP overexpression almost completely protected against HC loss in the apical turn, but this protective effect was not significant in the middle and basal turns. Thus, there might be other non-apoptotic mechanisms involved in $\mathrm{HC}$ death in the middle and basal turns as we discussed previously. 
All of these results showed that when neomycin was injected during the ototoxicity-sensitive period HC loss in the apical turn was primarily caused by hypersensitivity to neomycin-induced ototoxicity and that XIAP overexpression almost completely prevented this $\mathrm{HC}$ loss in the apical turn. We thus conclude that apoptotic factors are involved in the apical turn during the ototoxicity-sensitive period.

In contrast to the protective effect described above, XIAP overexpression failed to protect against hearing loss or against $\mathrm{HC}$ loss in middle and basal turns of the cochlea. Our qPCR data showed that Casp3 expression was significantly elevated in the entire cochlea when neomycin was injected during the ototoxicitysensitive period, but overexpression of XIAP-the most potent caspase inhibitor-was only able to partially prevent HC loss in the middle and basal turns. One possible reason for this limited protection is that neomycin-induced ototoxicity in the middle and basal turns is not mediated by the classical caspase-induced apoptosis pathway or the JNK signaling pathway, but rather by caspase-independent apoptosis pathways (Jiang et al., 2006) or by necrosis due to the chronic nature of drug administration (Wang and Li, 2000). It is also possible that ubiquitous overexpression of human XIAP in transgenic mice might not be sufficient to protect against the neomycin-induced HC loss in the middle and basal turns where neomycin concentrations are higher.

In summary, our results show that XIAP overexpression can successfully prevent neomycin-induced hearing loss and $\mathrm{HC}$ loss in the apical turn of the cochlea during the sensitive period. These results also provide in vivo evidence that apoptotic factors are involved in the sensitive period of neomycin-induced ototoxicity. Overexpression of XIAP, therefore, might serve as a novel therapeutic target for reducing aminoglycoside-induced HC damage and hearing loss.

\section{AUTHOR CONTRIBUTIONS}

Shan Sun, Jian Wang, Shankai Yin, Renjie Chai and Huawei Li developed the concepts or approach; Shan Sun, Mingzhi Sun, Yanping Zhang, Cheng Cheng, Muhammad Waqas, Huiqian Yu, Yingzi He, and Bo Xu performed experiments; Shan Sun, Mingzhi Sun, Cheng Cheng, Muhammad Waqas, Lei Wang performed data analysis; Shan Sun, Mingzhi Sun, Lei Wang, Renjie Chai and Huawei Li wrote the manuscripts, Yanping Zhang, Cheng Cheng, Muhammad Waqas, Huiqian Yu, Yingzi He, Bo Xu, Jian Wang and Shankai Yin edited the manuscript.

\section{ACKNOWLEDGMENTS}

The authors wish to thank Jin Li and Yalin Huang for technical assistance with confocal microscopy and Wenli Ni and Yusu Ni for breeding mice and providing technical support with mouse genotyping. This work was supported by grants from the Major State Basic Research Development Program of China (973 Program) (2011CB504500, 2010CB945503), the National Natural Science Foundation of China (Nos. 81371094, 81230019, 81100826, 81470692), the Shanghai Rising-Star Program (12QA1400500) Jiangsu Province Natural Science Foundation, BK20140620, the Specialized Research Fund for the Doctor Program of Higher Education (20120071110077), the Program of Leading Medical
Personnel in Shanghai, and the Fundamental Research Funds for the Central Universities (2242014R30022, NO2013WSN085).

\section{SUPPLEMENTARY MATERIAL}

The Supplementary Material for this article can be found online at: http://www.frontiersin.org/journal/10.3389/fncel. 2014.00248/abstract

Figure S1 | Caspase-3 expression in WT mice after neomycin treatment. (A) Representative confocal images of myo7a immunofluorescence in cochlear whole mounts of WT mice sacrificed at 1,3,5, or 7 days after the last injection of neomycin or saline (control). Neomycin or saline was injected between $P 1$ and $P 7$ (Group 1), P8 and P14 (Group 2), P15 and P21 (Group 3), or P60 and P66 (Group 4). (B) Myo7a+ HC quantification in WT mice treated with neomycin or saline (control) between P1 and P7 (Group 1), $P 8$ and P14 (Group 2), P15 and P21 (Group 3), or P60 and P66 (Group 4) and sacrificed at $1,3,5$, or 7 days after the last injection of neomycin or saline. ${ }^{* *} p<0.01$ vs. control and ${ }^{\#} p<0.05,{ }^{\# \#} p<0.01$ vs. Group 2, $n=3$. Scale bar in $\mathbf{A}=10 \mu \mathrm{m}$.

\section{REFERENCES}

Ashkenazi, A., and Dixit, V. M. (1998). Death receptors: signaling and modulation. Science 281, 1305-1308. doi: 10.1126/science.281.5381.1305

Berard, E., Garraffo, R., Chanalet, L., Dageville, C., Boutte, P., and Mariani, R. (1994). [Pharmacokinetics of netilmicin in the first use in newborn infants with gestational ages greater than 34 weeks]. Arch. Pediatr. 1, 481-488.

Chen, C. S., and Aberdeen, G. C. (1981). The sensitive period for induction of susceptibility to audiogenic seizures by kanamycin in mice. Arch. Otorhinolaryngol. 232, 215-220. doi: 10.1007/BF00457444

Chen, C. S., and Saunders, J. C. (1983). The sensitive period for ototoxicity of kanamycin in mice: morphological evidence. Arch. Otorhinolaryngol. 238, 217-223. doi: 10.1007/BF00453932

Chen, J. M., Williamson, P. A., Hutchin, T., Nedzelski, J. M., and Cortopassi, G. A. (1996). Topical gentamicin-induced hearing loss: a mitochondrial ribosomal RNA study of genetic susceptibility. Am. J. Otol. 17, 850-852.

Cooper, L. B., Chan, D. K., Roediger, F. C., Shaffer, B. R., Fraser, J. F., Musatov, S., et al. (2006). AAV-mediated delivery of the caspase inhibitor XIAP protects against cisplatin ototoxicity. Otol. Neurotol. 27, 484-490. doi: 10.1097/00129492-200606000-00009

Crocker, S. J., Liston, P., Anisman, H., Lee, C. J., Smith, P. D., Earl, N., et al. (2003). Attenuation of MPTP-induced neurotoxicity and behavioural impairment in NSE-XIAP transgenic mice. Neurobiol. Dis. 12, 150-161. doi: 10.1016/S09699961(02)00020-7

Cunningham, L. L., Cheng, A. G., and Rubel, E. W. (2002). Caspase activation in hair cells of the mouse utricle exposed to neomycin. J. Neurosci. 22, 8532-8540.

Debatin, K. M., and Krammer, P. H. (2004). Death receptors in chemotherapy and cancer. Oncogene 23, 2950-2966. doi: 10.1038/sj.onc.1207558

Deveraux, Q. L., and Reed, J. C. (1999). IAP family proteins-suppressors of apoptosis. Genes Dev. 13, 239-252. doi: 10.1101/gad.13.3.239

Deveraux, Q. L., Stennicke, H. R., Salvesen, G. S., and Reed, J. C. (1999). Endogenous inhibitors of caspases. J. Clin. Immunol. 19, 388-398. doi: 10.1023/A:1020502800208

Ding, D. L., and Salvi, R. (2007). Experiences in ototoxic investigations in aminoglycoside antibiotics. Chin. J. Otol. 5, 125. doi: 10.3969/j.issn.16722922.2007.02.001

Eggermont, J. J. (1986). Defining and determining sensitive periods. Acta Otolaryngol. Suppl. 429, 5-9. doi: 10.3109/00016488609122723

Emamaullee, J. A., Rajotte, R. V., Liston, P., Korneluk, R. G., Lakey, J. R., Shapiro, A. M., et al. (2005). XIAP overexpression in human islets prevents early posttransplant apoptosis and reduces the islet mass needed to treat diabetes. Diabetes 54, 2541-2548. doi: 10.2337/diabetes.54.9.2541

Forge, A. (1985). Outer hair cell loss and supporting cell expansion following chronic gentamicin treatment. Hear. Res. 19, 171-182. doi: 10.1016/03785955(85)90121-2 
Forge, A., and Li, L. (2000). Apoptotic death of hair cells in mammalian vestibular sensory epithelia. Hear. Res. 139, 97-115. doi: 10.1016/S0378-5955(99) 00177-X

Guo, Y., Jiang, S., Yang, W., and Han, D. (2001). [Experimental study on polyaspartic acid inhibition of gentamicin-induced generation of reactive oxygen species in guinea pig cochlea]. Zhonghua Er Bi Yan Hou Ke Za Zhi 36, 267-270. doi: 10.3760/j.issn:1673-0860.2001.04.009

Henley, C. M., and Rybak, L. P. (1993). Developmental ototoxicity. Otolaryngol. Clin. North Am. 26, 857-871.

Henley, C. M., and Rybak, L. P. (1995). Ototoxicity in developing mammals. Brain Res. Brain Res. Rev. 20, 68-90. doi: 10.1016/0165-0173(94)00006-B

Henley, C. M., Weatherly, R. A., Martin, G. K., and Lonsbury-Martin, B. (1996). Sensitive developmental periods for kanamycin ototoxic effects on distortionproduct otoacoustic emissions. Hear. Res. 98, 93-103. doi: 10.1016/03785955(96)00077-9

Hu, J., Du, J., Zhang, L., Price, S. R., Klein, J. D., and Wang, X. H. (2010). XIAP reduces muscle proteolysis induced by CKD. J. Am. Soc. Nephrol. 21, 1174-1183. doi: 10.1681/ASN.2009101011

Ishigaki, S., Liang, Y., Yamamoto, M., Niwa, J., Ando, Y., Yoshihara, T., et al. (2002). $\mathrm{X}$-Linked inhibitor of apoptosis protein is involved in mutant SOD1-mediated neuronal degeneration. J. Neurochem. 82, 576-584. doi: 10.1046/j.14714159.2002.00998.x

Jensen-Smith, H. C., Hallworth, R., and Nichols, M. G. (2012). Gentamicin rapidly inhibits mitochondrial metabolism in high-frequency cochlear outer hair cells. PLoS ONE 7:e38471. doi: 10.1371/journal.pone.0038471

Jiang, H., Sha, S. H., Forge, A., and Schacht, J. (2006). Caspase-independent pathways of hair cell death induced by kanamycin in vivo. Cell Death Differ. 13, 20-30. doi: 10.1038/sj.cdd.4401706

Jiang, X., and Wang, X. (2004). Cytochrome C-mediated apoptosis. Annu. Rev. Biochem. 73, 87-106. doi: 10.1146/annurev.biochem.73.011303.073706

Kaiser, C. L., Chapman, B. J., Guidi, J. L., Terry, C. E., Mangiardi, D. A., and Cotanche, D. A. (2008). Comparison of activated caspase detection methods in the gentamicin-treated chick cochlea. Hear. Res. 240, 1-11. doi: 10.1016/j.heares.2008.03.003

Li, L., Nevill, G., and Forge, A. (1995). Two modes of hair cell loss from the vestibular sensory epithelia of the guinea pig inner ear. J. Comp. Neurol. 355, 405-417. doi: 10.1002/cne.903550307

Matsui, J. I., Ogilvie, J. M., and Warchol, M. E. (2002). Inhibition of caspases prevents ototoxic and ongoing hair cell death. J. Neurosci. 22, 1218-1227.

Nadol, J. B. Jr. (1993). Hearing loss. N. Engl. J. Med. 329, 1092-1102. doi: 10.1056/NEJM199310073291507

Nakagawa, T., Yamane, H., Shibata, S., and Nakai, Y. (1997). Gentamicin ototoxicity induced apoptosis of the vestibular hair cells of guinea pigs. Eur. Arch. Otorhinolaryngol. 254, 9-14. doi: 10.1007/BF02630749

Plesner, A., Soukhatcheva, G., Korneluk, R. G., and Verchere, C. B. (2010). XIAP inhibition of beta-cell apoptosis reduces the number of islets required to restore euglycemia in a syngeneic islet transplantation model. Islets 2, 18-23. doi: 10.4161/isl.2.1.9997

Salvesen, G. S., and Riedl, S. J. (2008). Caspase mechanisms. Adv. Exp. Med. Biol. 615, 13-23. doi: 10.1007/978-1-4020-6554-5_2

Scaglione, F., Vigano, A., Colucci, R., Zampaglione, N., Lin, C. C., Cutler, D., et al. (1995). Pharmacokinetics of isepamicin in paediatric patients. J. Chemother. 7(Suppl. 2), 63-69.

Sha, S. H., Taylor, R., Forge, A., and Schacht, J. (2001). Differential vulnerability of basal and apical hair cells is based on intrinsic susceptibility to free radicals. Hear. Res. 155, 1-8. doi: 10.1016/S0378-5955(01)00224-6

Shiozaki, E. N., Chai, J., Rigotti, D. J., Riedl, S. J., Li, P., Srinivasula, S. M., et al. (2003). Mechanism of XIAP-mediated inhibition of caspase-9. Mol. Cell 11, 519-527. doi: 10.1016/S1097-2765(03)00054-6

Srinivasula, S. M., Hegde, R., Saleh, A., Datta, P., Shiozaki, E., Chai, J., et al. (2001). A conserved XIAP-interaction motif in caspase- 9 and Smac/DIABLO regulates caspase activity and apoptosis. Nature 410, 112-116. doi: 10.1038/35065125

Sugahara, K., Rubel, E. W., and Cunningham, L. L. (2006). JNK signaling in neomycin-induced vestibular hair cell death. Hear. Res. 221, 128-135. doi: 10.1016/j.heares.2006.08.009
Suzuki, Y., Nakabayashi, Y., Nakata, K., Reed, J. C., and Takahashi, R. (2001). $\mathrm{X}$-linked inhibitor of apoptosis protein (XIAP) inhibits caspase-3 and -7 in distinct modes. J. Biol. Chem. 276, 27058-27063. doi: 10.1074/jbc.M10 2415200

Tabuchi, K., Pak, K., Chavez, E., and Ryan, A. F. (2007). Role of inhibitor of apoptosis protein in gentamicin-induced cochlear hair cell damage. Neuroscience 149, 213-222. doi: 10.1016/j.neuroscience.2007.06.061

Trapp, T., Korhonen, L., Besselmann, M., Martinez, R., Mercer, E. A., and Lindholm, D. (2003). Transgenic mice overexpressing XIAP in neurons show better outcome after transient cerebral ischemia. Mol. Cell. Neurosci. 23, 302-313. doi: 10.1016/S1044-7431(03)00013-7

Unsain, N., Higgins, J. M., Parker, K. N., Johnstone, A. D., and Barker, P. A. (2013). XIAP regulates caspase activity in degenerating axons. Cell Rep. 4, 751-763. doi: 10.1016/j.celrep.2013.07.015

Wang, J., Menchenton, T., Yin, S., Yu, Z., Bance, M., Morris, D. P., et al. (2010). Over-expression of X-linked inhibitor of apoptosis protein slows presbycusis in C57BL/6J mice. Neurobiol. Aging 31, 1238-1249. doi: 10.1016/j.neurobiolaging.2008.07.016

Wang, J., Tymczyszyn, N., Yu, Z., Yin, S., Bance, M., and Robertson, G. S. (2011). Overexpression of X-linked inhibitor of apoptosis protein protects against noise-induced hearing loss in mice. Gene Ther. 18, 560-568. doi: $10.1038 /$ gt. 2010.172

Wang, Z., and Li, H. (2000). Microglia-like cells in rat organ of Corti following aminoglycoside ototoxicity. Neuroreport 11, 1389-1393. doi: 10.1097/00001756200005150-00008

Wei, X., Zhao, L., Liu, J., Dodel, R. C., Farlow, M. R., and Du, Y. (2005). Minocycline prevents gentamicin-induced ototoxicity by inhibiting p38 MAP kinase phosphorylation and caspase 3 activation. Neuroscience 131, 513-521. doi: 10.1016/j.neuroscience.2004.11.014

Xu, D., Bureau, Y., McIntyre, D. C., Nicholson, D. W., Liston, P., Zhu, Y., et al. (1999). Attenuation of ischemia-induced cellular and behavioral deficits by X chromosome-linked inhibitor of apoptosis protein overexpression in the rat hippocampus. J. Neurosci. 19, 5026-5033.

Ylikoski, J., Xing-Qun, L., Virkkala, J., and Pirvola, U. (2002). Blockade of cJun N-terminal kinase pathway attenuates gentamicin-induced cochlear and vestibular hair cell death. Hear. Res. 166, 33-43. doi: 10.1016/S0378-5955(01) 00388-4

Zhu, C., Xu, F., Fukuda, A., Wang, X., Fukuda, H., Korhonen, L., et al. (2007). $\mathrm{X}$ chromosome-linked inhibitor of apoptosis protein reduces oxidative stress after cerebral irradiation or hypoxia-ischemia through up-regulation of mitochondrial antioxidants. Eur. J. Neurosci. 26, 3402-3410. doi: 10.1111/j.14609568.2007.05948.x

Zou, H., Yang, R., Hao, J., Wang, J., Sun, C., Fesik, S. W., et al. (2003). Regulation of the Apaf-1/caspase-9 apoptosome by caspase-3 and XIAP. J. Biol. Chem. 278, 8091-8098. doi: 10.1074/jbc.M204783200

Conflict of Interest Statement: The authors declare that the research was conducted in the absence of any commercial or financial relationships that could be construed as a potential conflict of interest.

Received: 23 June 2014; paper pending published: 21 July 2014; accepted: 05 August 2014; published online: 15 September 2014.

Citation: Sun S, Sun M, Zhang Y, Cheng C, Waqas M, Yu H, He Y, Xu B, Wang L, Wang J, Yin S, Chai R and Li H (2014) In vivo overexpression of X-linked inhibitor of apoptosis protein protects against neomycin-induced hair cell loss in the apical turn of the cochlea during the ototoxic-sensitive period. Front. Cell. Neurosci. 8:248. doi: 10.3389/fncel.2014.00248

This article was submitted to the journal Frontiers in Cellular Neuroscience. Copyright (c) 2014 Sun, Sun, Zhang, Cheng, Waqas, Yu, He, Xu, Wang, Wang, Yin, Chai and Li. This is an open-access article distributed under the terms of the Creative Commons Attribution License (CC BY). The use, distribution or reproduction in other forums is permitted, provided the original author(s) or licensor are credited and that the original publication in this journal is cited, in accordance with accepted academic practice. No use, distribution or reproduction is permitted which does not comply with these terms. 